\title{
Plants with purple abaxial leaves: A repository of metrics from stomata distribution.
}

\author{
Humberto A. Filho' ${ }^{1}$, Odemir M. Bruno ${ }^{2}$, \\ São Carlos Institute of Physics, University of São Paulo, São Carlos - SP, \\ PO Box 369, 13560-970, Brazil. bruno@ifsc.usp.br
}

\begin{abstract}
Plants with purple abaxial leaf surfaces are very common in nature but the ecophysiological aspects of this phenotype are not well known. We have shown that the purple color of the abaxial surfaces generates an interesting contrast between the color of the stomata arranged on the epidermis, generally green, and the purple color of the pavement. This contrast makes the stomata completely visible to optic microscopy of the extant plants. This phenomenon made possible the proposition of a strategy to measure the distance between the stomata of the layer. The measurement of the distance between stomata generates accurate information of the distribution of stomata on the epidermis of living purple plants. In future ecophysiological inferences will be established from the information brought about by the measurements of the distance between stomata in purple plants.
\end{abstract}

\section{Introduction}

2 A purple coloration of lower abaxial leaf surfaces is commonly observed in 3 deeply-shaded understorey plants, especially in the tropics. However, the functional 4 significance to pigmentation, including its role in photosynthetic adaptation, remains 5 unclear [15. The pigments responsible for the coloring are known by anthocyanins, it can be found in plant species across a broad range of habitats, especially common in understoreyplants of the tropics [10 16] The leaf coloration of the abaxial surface may be transient or permanent depending of environmental conditions and plant species, [16]. Although the distribution of abaxial coloration among tropical taxa are widespread, it is not known the physiological significance of this phenotype. Some studies regarding the ecophysiological relationships involved in the foliar anthocyanin in upper adaxial surface have demonstrated that pigmentation play a rule in light-attenuation, protecting underlying cells from photoinhibition through the absorption of high energy blue-green wavelengths $[7,12$. Photoprotection have also been implicated in the physiological rules played by anthocyanins in plants in which exposed abaxial leaf surfaces are vulnerable to high-incident light. 14 Some studies have observed that the purple abaxial leaf surfaces appeared to reflect more red light than green surfaces. 17]. This observation induces the hypothesis that anthocyanin pigments may function to reflect adaxially-transmitted red light back into the mesophyll, in order to capture red photons in environments where light is limiting. This hypothesis is known as back-scatter propagation, it has not yet received a complete experimental validation but could explain the color of leaves in understorey plants. It is known that the abaxial surface of 
leaves some species of purple plants presents a high concentration of green stomatal cells which generates an interesting contrast between the color of the floor of the epidermis (purple) and the stomata (green), making this cells completely visible in studies of density and distribution pattern on the leave epidermis. 8 . The stomatic density varies according to the environmental light exposure. [11], then the color of the epidermis may also influence the distribution of stomata. In the current study we evaluate measures of distance between stomata at epidermis of purple plants as a derived parameter of stomatic density, through a automated method. Moreover we describe the variation of this parameter in 6 species of known purple plants: Tradescantia pallida, Tradescantia zebrina, Tradescantia minima, Callisia repens, Ctenante oppenheimiana Oxalis atropurpurea. Moreove the whole plant and microscopic images of 6 different types of Brazilian purple plants of species not known or not described in this work, are also provided for future studies.

\section{Materials and Methods}

\section{Plant material}

Purple plants of species not known had their collection sites referenced and a photograph of the whole plant was collected. The leaves of each plant were analyzed by optical microscopy. The unknown purple plants were named with the codes USP1, USP2, USP3, USP4, USP5, USP6 for proper assignment. Cuttings of $0.5 \mathrm{~cm}^{2}$ of the leaves of each plant were put in slides, with the abaxial surface facing up, covered with water, over covered with coverslips, and submitted to optic microscopy at microscope Axio-Lab A1-Zeiss at magnifications $50 \times$ and $100 \times$.

Geographical coordinates, microscopic and whole plant images are available at the following web addresses:

10 USP1- https ://dataverse .harvard.edu/dataset .xhtml?persistentId=doi : 10.7910/DVN/YSLJRW $[3]$

USP2- https://dataverse.harvard.edu/dataset.xhtml?persistentId=doi: 10.7910/DVN/VYMSUX 5

USP3-https://dataverse.harvard.edu/dataset .xhtml?persistentId=doi: 10.7910/DVN/DW3SNJ 4$]$

USP4-https://dataverse. harvard.edu/dataset .xhtml?persistentId=doi : 10.7910/DVN/V8GZYZ 6 ]

USP5-https://dataverse . harvard.edu/dataset . xhtml?persistentId=doi : 10.7910/DVN/CJCVZS 2

USP6- https://dataverse. harvard.edu/dataset. xhtml?persistentId=doi : 10.7910/DVN/6GYAGO 1$]$

Samples of $0.5 \mathrm{~cm}^{2}$ of the leaves of the species Tradescantia pallida,Tradescantia zebrina and Ctenante oppenheimiana, were also submitted to optic microscopy. The same procedure was applied to whole leaves from plants Tradescantia minima, Callisia repens, and Oxalis atropurpurea. 


\section{Stomata morphometry}

The stomata from a microscopic image of the abaxial surface from a purple leaf is manually assigned. The geometric center of each stomata is determined by an algorithm written in the matlab language, from which the cartesian coordinates of the centroid pixel $\left(x_{i}, y_{i}\right)$ of each point are obtained. The distance between each pair of points in the image is given by the equation below:

$$
d(i, j)=\sqrt{\left(x_{i}-x_{i+1}\right)^{2}+\left(y_{j}-y_{j+1}\right)^{2}}
$$

where $\mathrm{d}$ is the measure of the distance between two stomatal centroids $\left(x_{i}, y_{i}\right)$ and $(x(i+1), y(j+1))$. An array of distances between stomata of a generic image is then defined as shown in Figure 1 .

A)

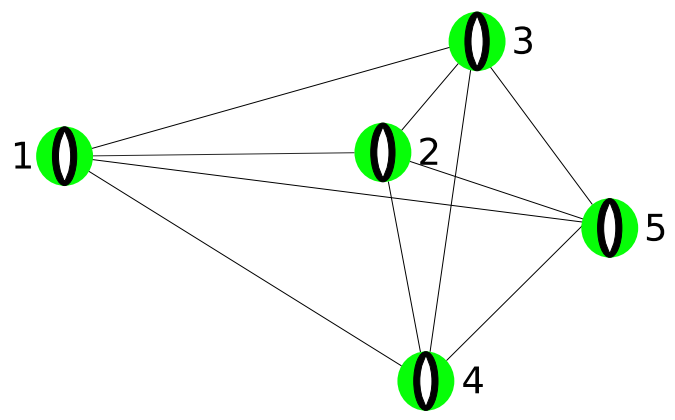

B)

\begin{tabular}{|c|c|c|c|c|c|}
\hline $\mathbf{0}$ & $\mathbf{1}$ & $\mathbf{2}$ & $\mathbf{3}$ & $\mathbf{4}$ & $\mathbf{5}$ \\
\hline $\mathbf{1}$ & 0 & $\mathrm{~d}_{12}$ & $\mathrm{~d}_{13}$ & $\mathrm{~d}_{14}$ & $\mathrm{~d}_{15}$ \\
\hline $\mathbf{2}$ & $\mathrm{d}_{21}$ & 0 & $\mathrm{~d}_{23}$ & $\mathrm{~d}_{24}$ & $\mathrm{~d}_{25}$ \\
\hline $\mathbf{3}$ & $\mathrm{d}_{31}$ & $\mathrm{~d}_{32}$ & 0 & $\mathrm{~d}_{34}$ & $\mathrm{~d}_{35}$ \\
\hline $\mathbf{4}$ & $\mathrm{d}_{41}$ & $\mathrm{~d}_{42}$ & $\mathrm{~d}_{43}$ & 0 & $\mathrm{~d}_{45}$ \\
\hline $\mathbf{5}$ & $\mathrm{d}_{51}$ & $\mathrm{~d}_{52}$ & $\mathrm{~d}_{53}$ & $\mathrm{~d}_{54}$ & 0 \\
\hline
\end{tabular}

Figure 1. A) Five stomata (green) connected by edges. B) Distance matrix of each pair of stomata, where $\mathrm{d}(\mathrm{i}, \mathrm{j})$ is the distance between the stomata drawn in A. 


\section{${ }_{72}$ Results}

\section{Model purple plants}

${ }_{74}$ We found a purple plant from specie Ctenante oppenheimiana with highly contrasting green stomata on the leaf epidermis. The Ctenante oppenheimiana plant can be used as a model plant in morphometric studies based on distances between stomata. The figure 2 shows an optical microscopy image from the abaxial epidermis of the plant and a model measurement based on distance between stomata neighbours. 2
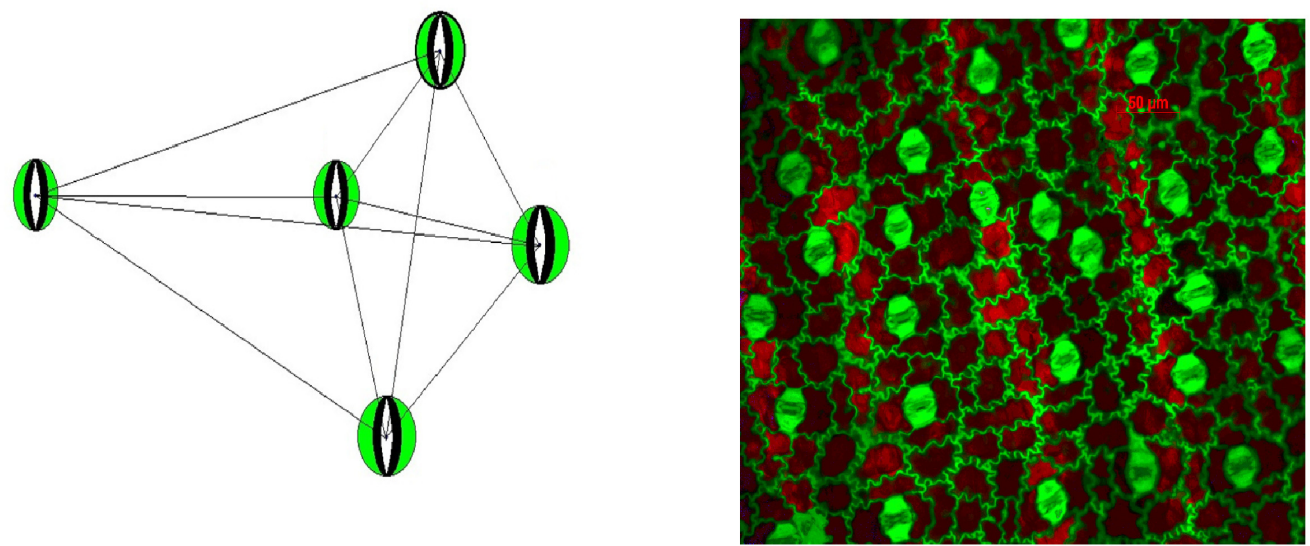

Figure 2. A) Connection network of the stomata neighbours, the edges represent the distance between stomata B) Optic microscopy from abaxial leaf from Ctenante oppenheimiana, magnification $200 \times$, highly contrasting green stomata and pavement cell wall.

Fully visible stomata contrasting with the purple epidermis were also found in 12 other species of plants with purple abaxial leaf, investigated in this work of which 6 are of known species. Microscopic and whole plant images for the 6 unknown species are available at [3, [5], [4, 6], 2], 1].

Samples of microscopic images of 6 other known plant species with purple abaxial leaf are shown in figure 3 The optical microscopy images obtained from Tradescantia pallida, Tradescantia zebrina, Callisia repens, Tradescantia minima, Ctenanthe oppenheimiana e Oxalis atropurpurea show stomata in extant leaves. 

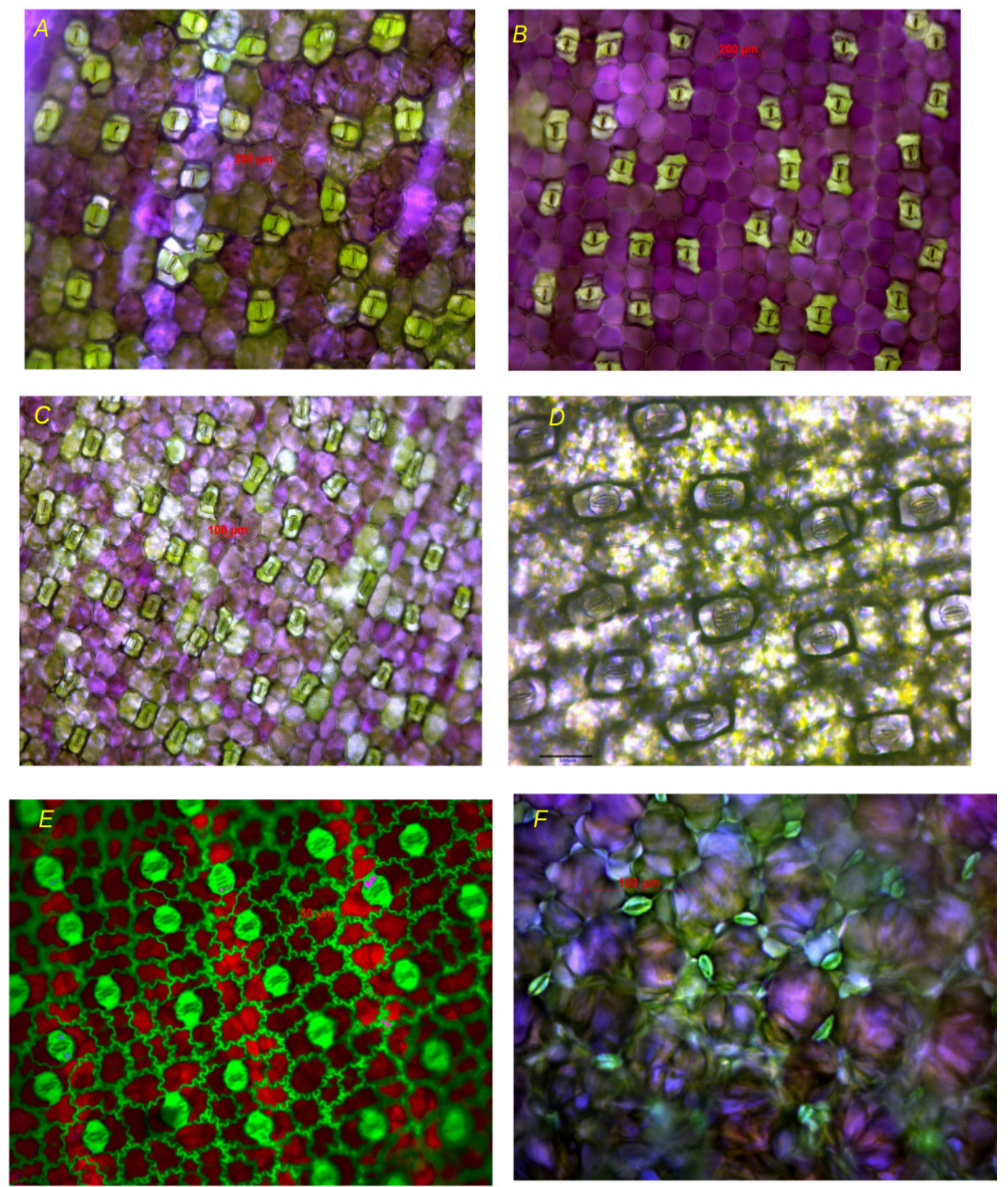

Figure 3. Optical microscopy images shown green stomata distributed over the abaxial surface of leaves. A) Image from Tradescantia pallida magnified 50× B) Image from Tradescantia zebrina magnified $50 \times$ C) Image from Callisia repens magnified $50 \times$ D) Image from Tradescantia minima magnified $100 \times$. E) Image from Ctenanthe oppenheimiana, magnified $200 \times$. F) Image from Oxalis atropurpurea magnified $200 \times$.

The microscopic magnification values used to collect the images of the Figure 3 give the dimension of the size of the stomata in the studied species. For example, the length of the stomata from Tradescantia pallida, the largest among all the plants studied, is on 
the order of more than 10 times the stomata of the plant Oxalis atropurpurea, the smallest.

Although all plants have the pavement color contrasting with the color of the stomata, the color of the abaxial face has plasticity, especially in the species Oxalis atropurpurea and Tradescantia minima in which they varied from green to purple according to environmental variations (Data not shown).

\subsection{Morphometrics}

It is noted that depending on the species and environmental condition, the size of the stomata may range from 10 to $100 \mu \mathrm{M}$ in length and occur at densities ranging from 5 to 1000 stomata per $\mathrm{mm}^{2}$. 9] In spite of this great variability, there is a general tendency in the relationship between stomatal density and stomata size for different plants, usually plants with high stomatal density, presenting small stomata and vice versa. Many studies have reported the inverse relationship between leaf stomata density and stomatal cell area 13 . We found the same relationship for the 6 species studied in this paper. The graph shown in Figure 4 quantifies the measurements.

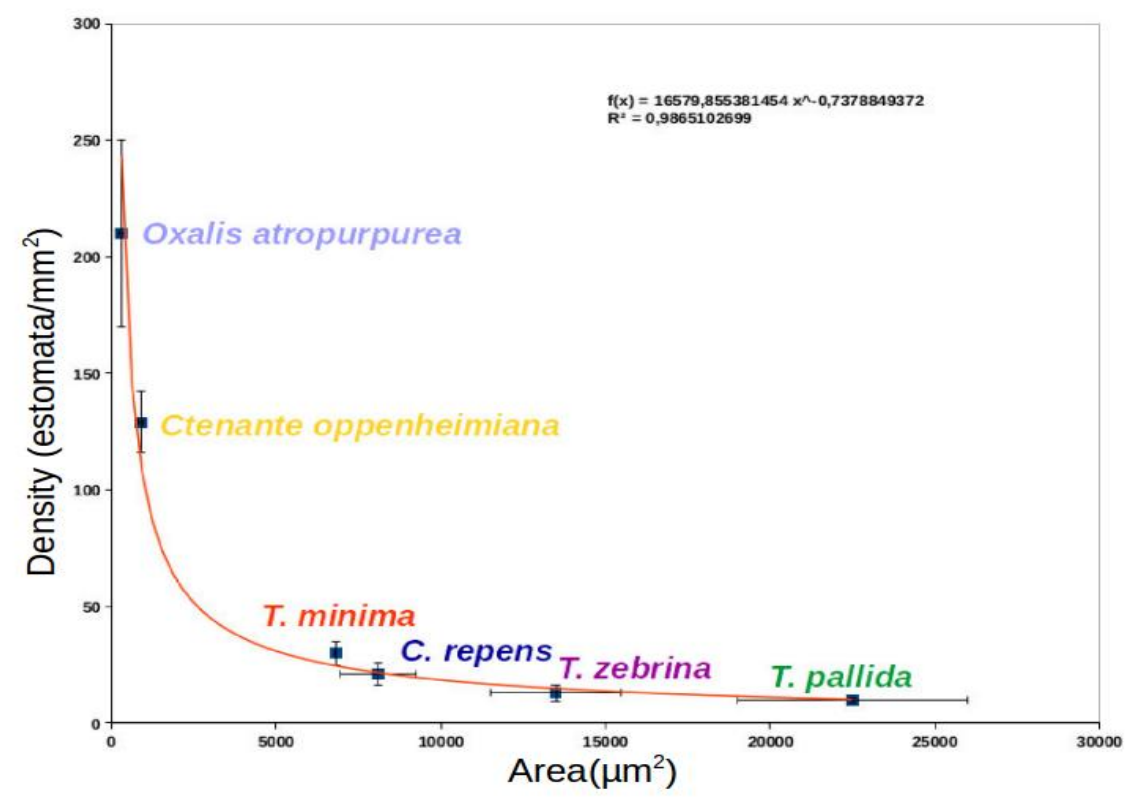

Figure 4. The graph shows the inverse relationship between mean stomata density and mean stomatic area. The plant T. pallida is the one with the largest stomata area and the lowest average density. Species indicated by name at each point in the graph. Equation of the graph showing geometric decay of the mean area of the stomata as a function of their densities in the images. The error bars show the standard deviations of the measures calculated from $\mathrm{n}=18$ images of 6 plants per species. 
The observation of the microscopic images from purple abaxial leaves, make full the observation of stomata in extant purple plants and generates the spatial inference that the distances between stomata vary in relation to their densities. The distance measurements of neighbor stomata quantify how the plasticity of the leaf can be measured from the distribution of these cells in the epidermis. The measurements of the mean distances between neighboring stomata relative to the stomatal densities can be evidenced in the graphs of Figure 5 which show the dispersion of the density values as a function of the mean distance between the stomata neighbors for the six species of purple plants. 
A)

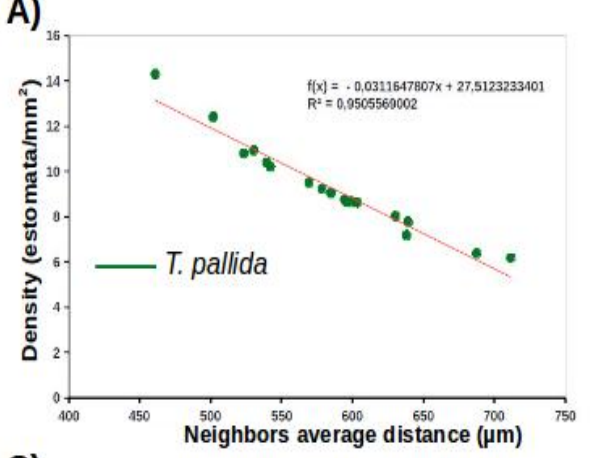

C)

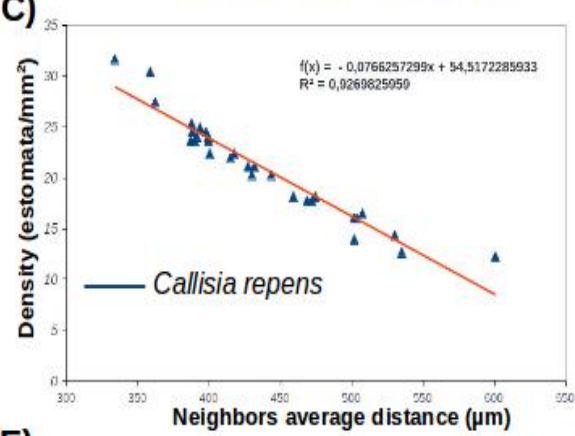

E)

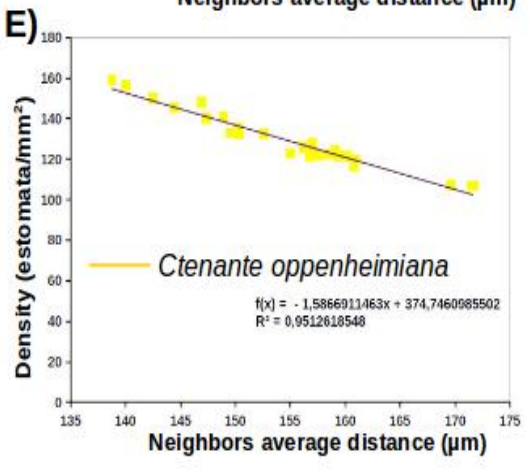

B)
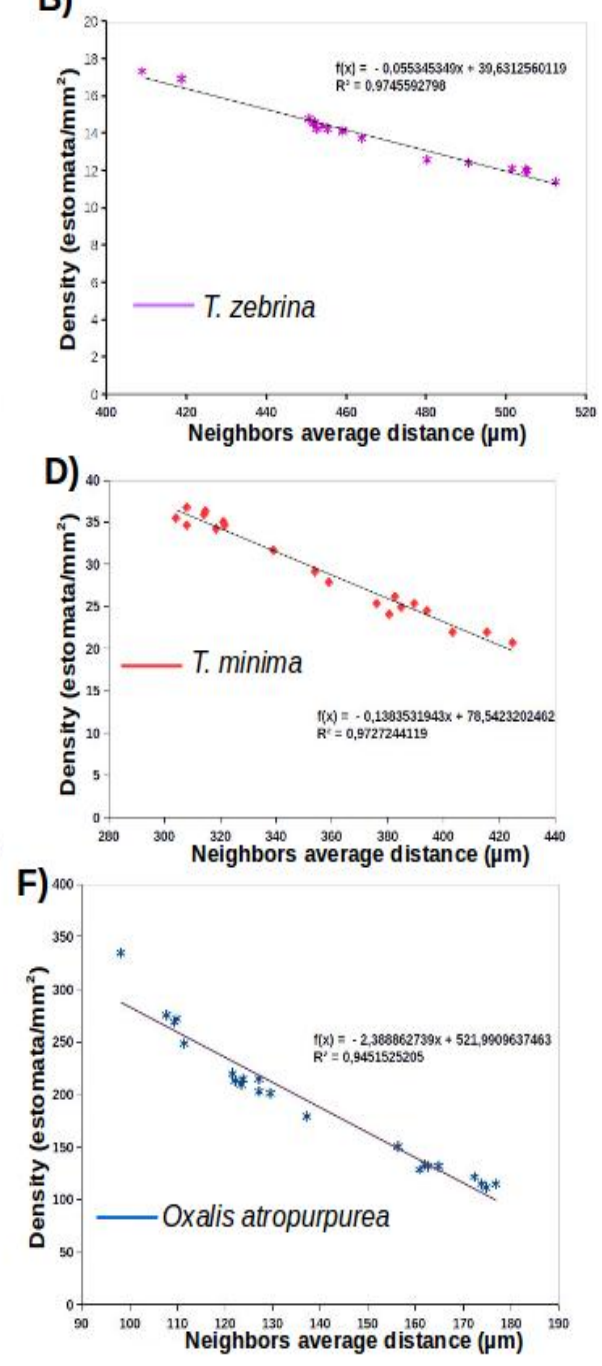

Figure 5. Dispersion of the mean distance values between neighboring stomata as a function of stomatal density for six plants, plots from top to bottom: A) T. pallida B) Tradescantia zebrina C) Callisia repens. D) T. minima E) Ctenanthe oppenheimiana. F) Oxalis atropurpurea. Cada ponto no gráfico representa uma medida em uma imagem. 
The results shown in Figure 5 indicate that the mean distances between neighboring stomata are inversely proportional to their stomatic densities and vary linearly therefrom in each species independently. These measurements are, therefore, direct evidence of plasticity as regards the variation of stomata distribution on leaf epidermis. When transposing the mean values of the measurements indicated in the graphs shown above, for a single graph that quantifies the relation distance $\times$ density between plant species, it can be observed a tendency of geometric decay of distances between stomata with respect to density. The graph showing this trend is shown in the Figure 6 .

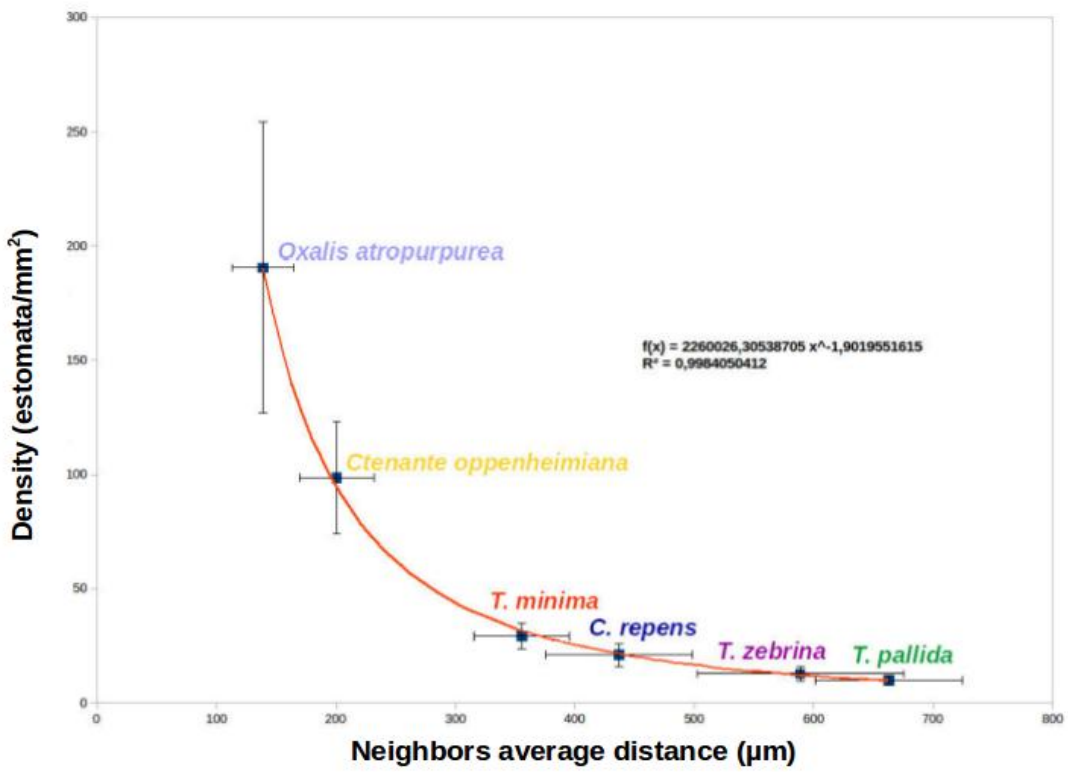

Figure 6. Dispersion of the mean distance from stomata neighbors as a function of the mean stomatic density for Tradescantia pallida, Tradescantia zebrina, Callisia repens, Tradescantia minima, Ctenanthe oppenheimiana and Oxalis atropurpurea. The error bars indicate the standard deviation of the measurements in relation to the mean values of distances between stomata and stomatal densities by species. ( $\mathrm{N}=5$ plants per species, 3 images per plant). 
It can be inferred from the graph of Figure 6 that the distance between stomata decays geometrically with the increase in stomatal density in plants of different species, the same trend to that shown for the density $\times$ area of the stomata seen earlier in Figure 4. This trend probably applies to any set of plants of different species with significant differences in mean stomatal densities.

The measures of distance between stomata have been little used as criterion of estimation of phenotypic plasticity in plants. The results shown here indicate that this measure quantifies the dispersion of the stomata and precisely measures its distribution on the leaf. Plants with a purple abaxial surface exhibit a natural color contrast with stomata, which are usually green due to the large amount of chlorophyll and chloroplasts present within the guard cell. Therefore the measurement of distance between stomata in purple abaxial surface plants, seems to be a powerful strategy for observation of changes in the physiology of plants, generated by changes in the environment. Thus ecophysiological aspects in plants will be approached more accurately from the estimation in distribution of stomata on the leaves. It is known that the stomata feel the environmental changes, being natural sensors of the plant the conditions of the environment, at the same time that they are responsible for the climate change. 13 However, it is not known exactly how the great amount of physical-chemical factors of the environment alters the distribution of stomata.

Thus the strategy of stomata morphometry in purple plants, shown in this paper, may provide valuable answers to the understanding of how plants respond to climate change.

\section{References}

1. H. Antunes de Almeida Filho. Amazing green stomata at purple abaxial surface leave of a unknown specie., 2018.

2. H. Antunes de Almeida Filho. Green stomata on the purple abaxial surface of the leaf from a dicotyledon plant found in the gardens of the usp-são carlos university., 2018.

3. H. Antunes de Almeida Filho. Purple dicot plant found at gardens of physics institute of são carlos -usp- brazil., 2018.

4. H. Antunes de Almeida Filho. Purple monocot plant, 2018.

5. H. Antunes de Almeida Filho. Purple monocot with large leaves, 2018.

6. H. Antunes de Almeida Filho. Stomata of a completely purple dicotyledonous plant found in the gardens of usp-são carlos, 2018.

7. L. Chalker-Scott. Environmental significance of anthocyanins in plant stress responses. Photochemistry and photobiology, 70(1):1-9, 1999.

8. H. A. de Almeida Filho, J. Machicao, and O. M. Bruno. Geometric plasticity at leaves from ctenanthe oppenheimiana probed by measure of distances between stomata. In Journal of Physics: Conference Series, volume 936, page 012094. IOP Publishing, 2017.

9. S. H. Eckerson. The number and size of the stomata. Botanical Gazette, 46(3):221-224, 1908.

10. W. Forsyth and N. Simmonds. A survey of the anthocyanins of some tropical plants. Proc. R. Soc. Lond. B, 142(909):549-564, 1954. 
11. A. Gay and R. Hurd. The influence of light on stomatal density in the tomato. New Phytologist, 75(1):37-46, 1975.

12. K. S. Gould, D. N. Kuhn, D. W. Lee, and S. F. Oberbauer. Why leaves are sometimes red. Nature, 378(6554):241, 1995.

13. A. M. Hetherington and F. I. Woodward. The role of stomata in sensing and driving environmental change. Nature, 424(6951):901, 2003.

14. N. M. Hughes and W. K. Smith. Attenuation of incident light in galax urceolata (diapensiaceae): concerted influence of adaxial and abaxial anthocyanic layers on photoprotection. American Journal of Botany, 94(5):784-790, 2007.

15. N. M. Hughes, T. C. Vogelmann, and W. K. Smith. Optical effects of abaxial anthocyanin on absorption of red wavelengths by understorey species: revisiting the back-scatter hypothesis. Journal of experimental botany, 59(12):3435-3442, 2008.

16. D. W. Lee and T. M. Collins. Phylogenetic and ontogenetic influences on the distribution of anthocyanins and betacyanins in leaves of tropical plants. International Journal of Plant Sciences, 162(5):1141-1153, 2001.

17. D. W. Lee, J. B. Lowry, and B. Stone. Abaxial anthocyanin layer in leaves of tropical rain forest plants: enhancer of light capture in deep shade. Biotropica, pages $70-77,1979$.

\section{Acknowledgments}

H.A.F. gratefully acknowledges the financial support of CNPq grant \#153137/2013-4. 